\title{
Cost-Effectiveness Analysis of Maintenance
}

\section{Olaparib in Patients with Metastatic Pancreatic Cancer and a Germline BRCAI/2 Mutation Based on the POLO Trial}

This article was published in the following Dove Press journal:

Cancer Management and Research

\author{
Mei Zhan ${ }^{1-3}$ \\ Hanrui Zheng ${ }^{1-3}$ \\ Yu Yang ${ }^{2,4}$ \\ Zhiyao $\mathrm{He}^{\prime}$ \\ Ting $X u\left(\mathbb{D}^{1,3}\right.$ \\ Qiu Li $\mathbb{1}^{2,4}$
}

'Department of Pharmacy, West China Hospital, Sichuan University, Chengdu, People's Republic of China; ${ }^{2}$ West China Biomedical Big Data Center, Sichuan University, Chengdu, People's Republic of China; ${ }^{3}$ West China School of Pharmacy, Sichuan University, Chengdu, People's Republic of China; ${ }^{4}$ Department of Medical Oncology, Cancer Center, West China Hospital, Sichuan University, Chengdu, People's Republic of China
Correspondence: Ting $\mathrm{Xu}$

Department of Pharmacy, West China Hospital, Sichuan University, Chengdu, Sichuan 61004I, People's Republic of China

Email tingx2009@163.com

Qiu Li

Department of Medical Oncology, Cancer Center, West China Hospital, Sichuan

University, Chengdu, Sichuan 6I004I,

People's Republic of China

Email fbqiu9@163.com
Objective: The phase III POLO trial demonstrated that olaparib as maintenance therapy for metastatic pancreatic cancer patients with a germline BRCA mutation had greater efficacy than placebo, but maintenance olaparib places an economic burden on patients. This study evaluated the cost-effectiveness of olaparib as maintenance therapy based on the POLO trial (NCT02184195).

Methods: A three-state Markov model (progression-free survival [PFS], progressive disease [PD] and death) based on data from the POLO trial was used to estimate the incremental cost-effectiveness ratio (ICER) of maintenance olaparib versus placebo for metastatic pancreatic cancer patients with a germline BRCA mutation. The cost was evaluated from the Chinese society's perspective, and health outcomes were assessed in terms of qualityadjusted life years (QALYs). The primary outcome was the ICER gained in terms of 2019 US\$ per QALY. Model robustness was explored with one-way and probabilistic sensitivity analyses.

Results: Compared with placebo, maintenance olaparib increased costs by $\$ 23,544.35$ while gaining 0.69 QALYs, resulting in an ICER of \$34,122.25 per QALY. The ICER was far higher than the commonly accepted willingness-to-pay threshold (\$28,255.55 per QALY).

Conclusion: Compared with placebo, maintenance olaparib for metastatic pancreatic cancer patients with a germline BRCA mutation is not cost-effective in China.

Keywords: cost effectiveness, pancreatic cancer, olaparib, BRCA, Markov model

\section{Introduction}

Worldwide, it was estimated that there were almost 458,918 new cases of pancreatic cancer and 432,242 associated deaths in 2018. ${ }^{1}$ The incidence of pancreatic cancer continued to increase from 1930 to $2017 .^{2}$ The survival rates for many cancer patients are improving because of new treatment or early detection, but the five-year survival rates of pancreatic cancer patients remain very low. ${ }^{3,4}$ It is predicted that pancreatic cancer will become the second leading cause of cancer-related death by 2030 in the United States..$^{5}$ Only 4 to $7 \%$ of patients with pancreatic cancer lack BRCA1 or BRCA2 activity because of a BRCA mutation. ${ }^{6-8}$ Olaparib is a polyadenosine diphosphate-ribose polymerase (PARP) inhibitor that has been used in patients with tumors with BRCA mutations, and maintenance olaparib has improved progression-free survival (PFS) in patients with ovarian cancer with a germline BRCA1/2 mutation. ${ }^{9,10}$ The 
POLO trial, which included 154 metastatic pancreatic cancer patients with a germline BRCA1 or BRCA2 mutation that had not progressed during at least 16 weeks of first-line platinum-based chemotherapy, demonstrated a significant 3.6-month improvement in PFS in the olaparib group compared with the placebo group (7.4 vs 3.8 months; $\mathrm{HR}=0.53$; $\mathrm{P}=0.004){ }^{11}$ Based on the POLO trial, the National Comprehensive Cancer Network (NCCN) recommended olaparib as maintenance therapy for patients with a germline BRCA1 or BRCA2 mutation after first-line chemotherapy. ${ }^{12}$

Although the POLO trial demonstrated a significant PFS benefit, olaparib is dramatically expensive for patients and insurance payers. As such, we sought to evaluate the cost-effectiveness of olaparib as maintenance therapy for pancreatic cancer patients with a germline BRCA1 or BRCA2 mutation based on the POLO trial from the perspective of Chinese society.

\section{Materials and Methods}

\section{Patients and Regimens}

Clinical information was derived from the multicenter, randomized, phase III POLO trial. ${ }^{11}$ Adult patients with metastatic pancreatic cancer and a germline BRCA1 or BRCA2 mutation were recruited for the POLO trial. When patients finished receiving at least 16 weeks of first-line platinumbased chemotherapy without disease progression, they were randomly assigned to receive $300 \mathrm{mg}$ olaparib twice daily as maintenance therapy or matching placebo until disease progression or the development of unacceptable toxic effects.

\section{Markov Model}

A Markov model was constructed using TreeAge software (TreeAge, Williamstown, MA, USA) to estimate the cost and quality-adjusted life years (QALYs) of olaparib and placebo. The Markov model had three health states: PFS, progressive disease (PD) and death. All simulated patients started in the PFS state and could move to the PD state or remain in the PFS state, and after entering the PD state, patients could only stay in the PD state or move to death. The model diagram is shown in Figure 1. A 1-month cycle length was adopted based on the time span of disease duration and progression. The 5-year survival rate for pancreatic cancer patients is less than $10 \%{ }^{3}$ Therefore, we used a 5 -year time horizon in the Markov model. A 3\% annual discount rate was used for costs and effectiveness.

\section{Survival Estimates and Utilities}

Kaplan-Meier survival curves and other clinical information were obtained from the POLO trial. Efficacy and grade 3-4 adverse events (AEs) related to costs collected from the POLO trial are shown in Table 1. The time and survival probabilities were obtained by digitizing the Kaplan-Meier curves of PFS and overall survival (OS) from the POLO trial using Web-Plot Digitizer. The Weibull distribution was fitted to the Kaplan-Meier data for PFS or OS and the detailed method described by Hoyle and Henley. ${ }^{13}$ The input parameters of the Weibull distribution are shown in Table 2. The survival curve simulation results are presented in Figure 2. We used the formula $S(t)=P(T \geq t)=\exp \left(-\lambda t^{\gamma}\right)$ to estimate the survival probability at time $\mathrm{t}$ and the formula $\mathrm{P}(\mathrm{t})=1-\exp$ $\left[\left(\lambda(t-1)^{\gamma}-\lambda t^{\gamma}\right]\right.$ to estimate the transition probability at a given cycle $t$. There was no significant difference between the olaparib group and the placebo in terms of health-related quality of life in the POLO trial, ${ }^{14}$ and we assumed that the health utility values were the same in both groups. Health utility values were adopted from a recently published study. The utility values of the PFS state, PD state and death were $0.81,0.73$ and 0 , respectively. $^{15}$ In the Markov model, QALY= $\sum_{1}^{\text {cycle number }} \sum_{1}^{\text {statenumber }}$ utility $($ state $) \times \operatorname{probability}($ state $)$.

\section{Cost Estimates}

Costs were estimated from the perspective of Chinese society. Total costs in our analysis included costs for

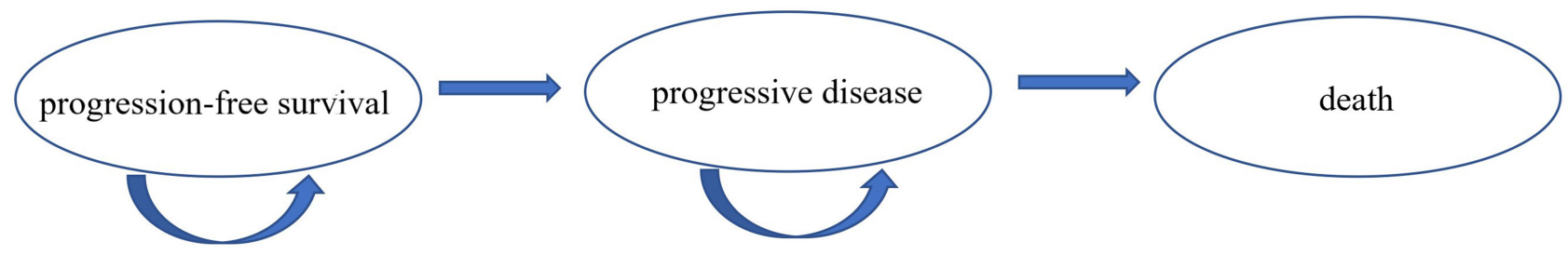

Figure I The Markov model simulated three health states: PFS, PD and death. Abbreviations: PFS, progression-free survival; PD, progressive disease. 
Table I Clinical Efficacy and Grade 3-4 Adverse Events

\begin{tabular}{|c|l|l|}
\hline \multirow{2}{*}{ Variables } & \multicolumn{2}{|l|}{ Base Case Value } \\
\cline { 2 - 3 } & $\begin{array}{l}\text { The Olaparib } \\
\text { Group }\end{array}$ & $\begin{array}{l}\text { The Placebo } \\
\text { Group }\end{array}$ \\
\hline Clinical efficacy & & \\
OS (Months) & 18.9 & 18.1 \\
PFS (Months) & 7.4 & 3.8 \\
\hline Probability of grade 3-4 AEs (\%) & & \\
Fatigue or asthenia & 5 & 2 \\
Nausea & 0 & 2 \\
Anemia & $1 \mathrm{I}$ & 3 \\
Abdominal pain & 2 & 2 \\
Decreased appetite & 3 & 0 \\
Vomiting & 1 & 2 \\
Back pain & 0 & 2 \\
Arthralgia & 1 & 0 \\
\hline
\end{tabular}

Abbreviations: OS, overall survival; PFS, progression-free survival; AEs, adverse event.

Table 2 Key Model Input Parameters

\begin{tabular}{|c|c|c|c|c|}
\hline \multirow{3}{*}{$\begin{array}{l}\text { Weibull survival model of } \\
\text { PFS of the Olaparib group }\end{array}$} & \multicolumn{4}{|l|}{ Parameter } \\
\hline & Intercept & 2.2734 & Lambda & 0.0964 \\
\hline & Log (scale) & 0.0287 & Gamma & 1.0291 \\
\hline \multirow{2}{*}{$\begin{array}{l}\text { Weibull survival model of } \\
\text { PFS of the Placebo group }\end{array}$} & Intercept & 1.6354 & Lambda & 0.0609 \\
\hline & Log (scale) & 0.5375 & Gamma & 1.7117 \\
\hline \multirow{2}{*}{$\begin{array}{l}\text { Weibull survival model of } \\
\text { OS of the Olaparib group }\end{array}$} & Intercept & 3.3695 & Lambda & 0.0158 \\
\hline & Log (scale) & 0.2076 & Gamma & 1.2308 \\
\hline \multirow{2}{*}{$\begin{array}{l}\text { Weibull survival model of } \\
\text { OS of the Placebo group }\end{array}$} & Intercept & 3.0509 & Lambda & 0.0046 \\
\hline & Log (scale) & 0.5689 & Gamma & 1.7663 \\
\hline
\end{tabular}

Abbreviations: PFS, progression-free survival; OS, overall survival.

treatment and AEs. The treatment costs included drugs, tests, and registration fees. The costs of drugs and tests were based on the 2019 fee standards of West China Hospital, Sichuan University. When olaparib is part of the treatment of pancreatic cancer, the donation policy is not applicable, so we did not consider donations when calculating the cost of olaparib. Only grade 3 or higher AEs that occurred in at least $1 \%$ of any group from the POLO trial were used to calculate the costs of AEs. We calculated the costs of hospitalization and absenteeism fees if the management of AEs required hospitalization. Details of the cost information are provided in Table 3. All costs were converted into US dollars, with an exchange rate of $\$ 1=¥ 6.8635$ (19 July 2019). We used the formula

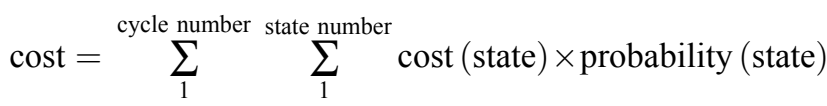
to calculate the total cost in the Markov model.

\section{Sensitivity Analysis}

One-way sensitivity analysis was conducted by varying key parameters within $\pm 20 \%$ of their baseline value individually to examine their impact on the results. A probabilistic sensitivity analysis was also performed to evaluate overall uncertainty in the optimal treatment strategies at different hypothetical willingness-to-pay (WTP) thresholds.

We set WTP at three times the gross domestic product (GDP) per capita, which was $\$ 28,255.55$ in China based on the World Health Organization (WHO) data. ${ }^{16}$ If the ICER was below $\$ 28,255.55 / \mathrm{QALY}$, interventions were defined as cost-effective.

\section{Results}

\section{Base Case Analysis}

In the base case (Table 2), the total costs of the olaparib group were $\$ 54,402.18$, and the total costs of the placebo group were $\$ 30,857.83$. The overall QALYs in the olaparib group were higher than those in the placebo group (1.91 QALYs vs 1.22 QALYs). The ICER of the olaparib group compared with the placebo group was $\$ 34,122.25$ per QALY, which was almost 2.7 times higher than the commonly accepted threshold for cost-effectiveness $(\$ 28,255.55$ per QALY in China).

\section{Sensitivity Analysis}

In the one-way sensitivity analysis, the cost after PD for both groups and the cost of olaparib were the most influential parameters in the model. The cost after PD in the placebo group had the highest impact on the ICER. In the placebo group, when the cost after PD varied from 1521.89 to 2282.83 , the ICER ranged from $\$ 25,797.76$ per QALY to $\$ 42,370.06$ per QALY. In addition, when the cost after PD in the olaparib group or the cost of olaparib was reduced by $20 \%$, the ICER was below WTP. However, changing other parameters, such as the utilities of PD or PFS, the duration of PFS or the costs of tests or AEs, did not decrease the ICER of olaparib into a range that would be considered cost-effective (Figure 3). The probabilistic sensitivity analysis showed that $0 \%$ of simulations showed olaparib to be cost-effective until WTP increased to above $\$ 35,000$ per QALY (Figure 4).

\section{Discussion}

In this study, we found that olaparib was not cost-effective as maintenance therapy for metastatic pancreatic cancer patients with a germline BRCA mutation compared with placebo. Seven patients in the placebo group received 


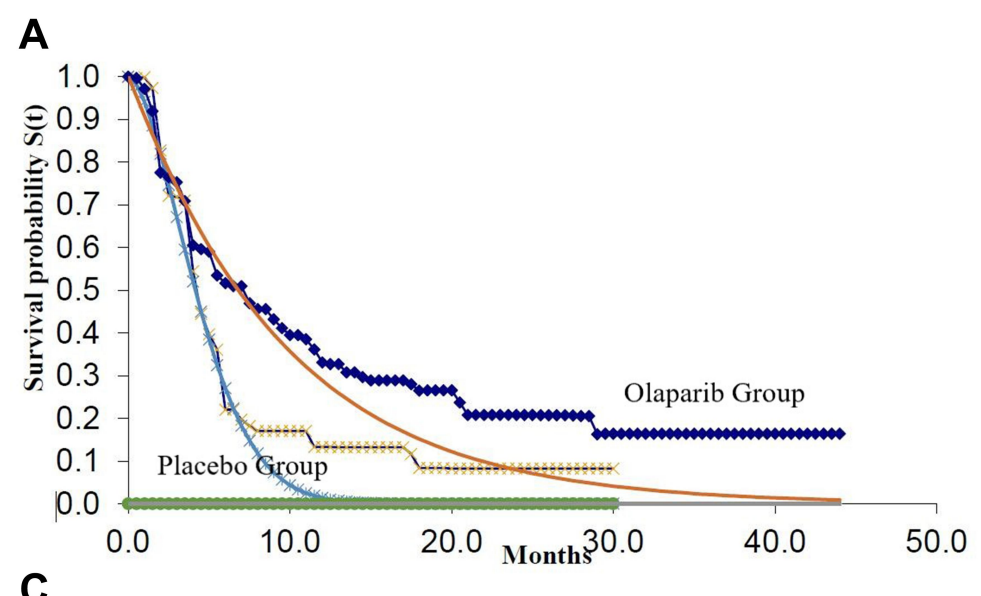

B
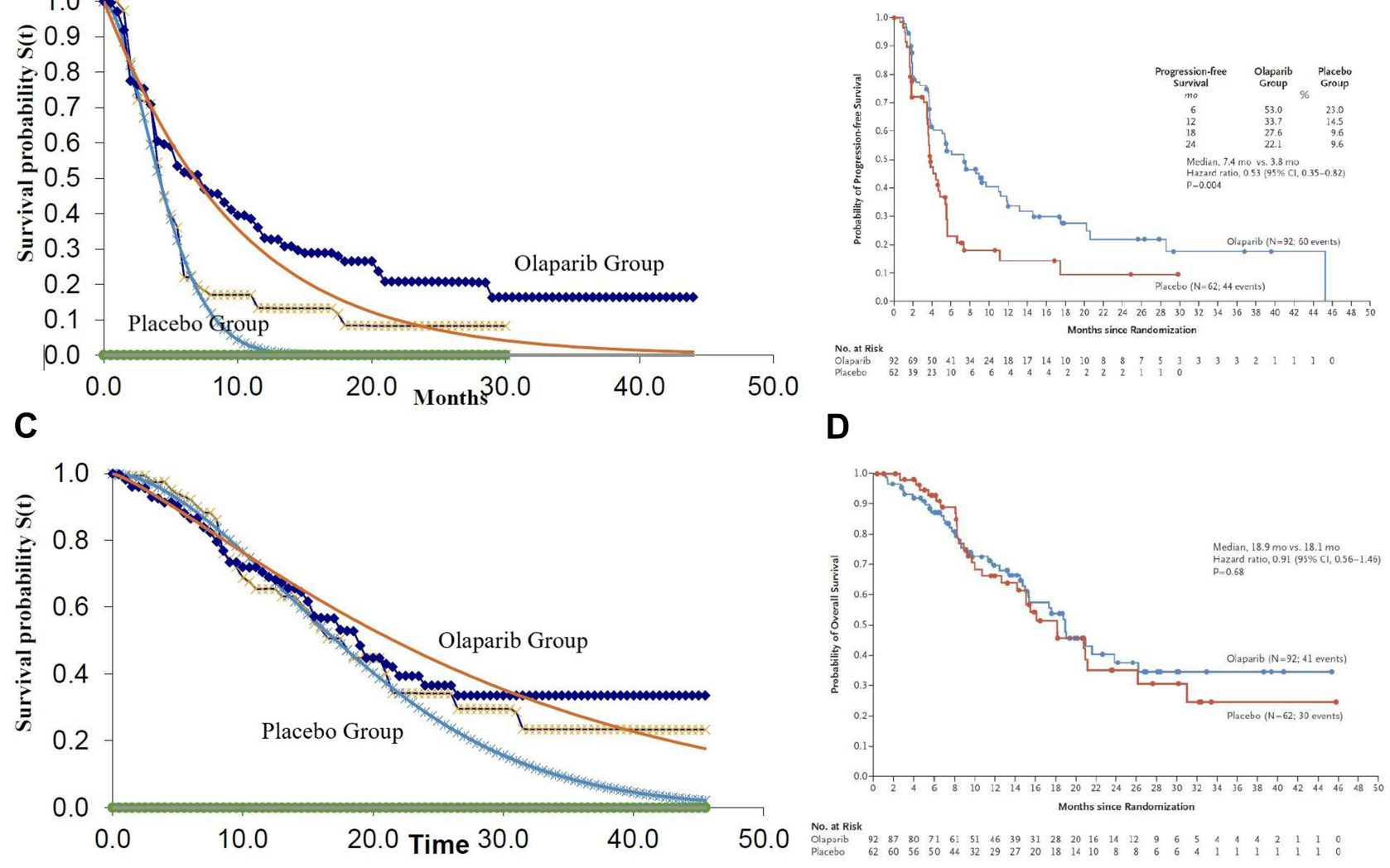

Figure 2 (A) Simulate overall survival curve for the Olaparib group and the Placebo group. (B) Simulate progression-free survival curve for the Olaparib group and the Placebo group. (C) Kaplan-Meier curve of overall survival from the POLO study. (D) Kaplan-Meier curve of the progression-free survival curve from the POLO study.

olaparib after PD as a subsequent therapy, so the price of olaparib had an impact on the cost of not only the PFS state but also the PD state. The price of olaparib decreased

Table 3 Results of the Cost-Effectiveness Analysis

\begin{tabular}{|c|l|l|}
\hline Parameters & $\begin{array}{l}\text { The } \\
\text { Olaparib } \\
\text { Group }\end{array}$ & $\begin{array}{l}\text { The } \\
\text { Placebo } \\
\text { Group }\end{array}$ \\
\hline Costs (\$) & $31,172.57$ & 2238.29 \\
PFS state (\$) & 2954.76 & 0.00 \\
cOlaparib (\$/month) & 8.08 & 0.56 \\
cAEs (\$/month) & 362.33 & 487.39 \\
cTest (\$/month) & $23,229.61$ & $28,619.53$ \\
PD state (\$) & $54,402.18$ & $30,857.83$ \\
Total Cost (\$) & $23,544.35$ & $/$ \\
Incremental costs (\$) & & \\
\hline Effectiveness (QALYs) & 0.63 & 0.30 \\
PFS state (QALYs) & 1.28 & 0.92 \\
PD state (QALYs) & 1.91 & 1.22 \\
Total effectiveness (QALYs) & 0.69 & $/$ \\
Incremental effectiveness (QALYs) & $34,122.25$ & \\
\hline ICERs compared with PC alone (\$/QALY) & & \\
\hline
\end{tabular}

Abbreviations: PFS, progression-free survival; PD, progressive disease; $A E$, adverse event; QALY, quality-adjusted life years; ICER, incremental cost-effectiveness ratio. from $\$ 7739.7$ per month to $\$ 2954.76$ per month at the beginning of 2020, and the ICER decreased from $\$ 97,416.84$ per QALY to $\$ 34,122.25$ per QALY. However, the ICER was still higher than WTP.

In addition to pancreatic cancer, olaparib was also not cost-effective when used in patients with ovarian cancer in the USA or breast cancer in Japan. ${ }^{17,18}$ In 2015, Smith built decision analysis models and found that the ICER was $\$ 258,864$ per progression-free life-year saved for olaparib maintenance therapy in ovarian cancer patients with a BRCA mutation. Drug cost had the highest impact on the ICER; to achieve an ICER of less than $\$ 50,000$, the cost of olaparib would have had to decrease to $\$ 2500$ or less per month. In addition, for wild-type BRCA1/2 patients, the ICER was $\$ 600,552$ per progression-free life-year saved. In 2019, Saito created a Markov cohort model and found that the ICER for olaparib monotherapy in metastatic breast cancer patients with BRCA1/2 mutations was $\$ 131,047 /$ QALY compared with standard chemotherapy alone.

Because of the assistance programs for olaparib in ovarian cancer, patients with ovarian cancer can buy a one-month supply of olaparib and obtain a one month supply for free. 


\section{Tornado Analysis (ICER)}

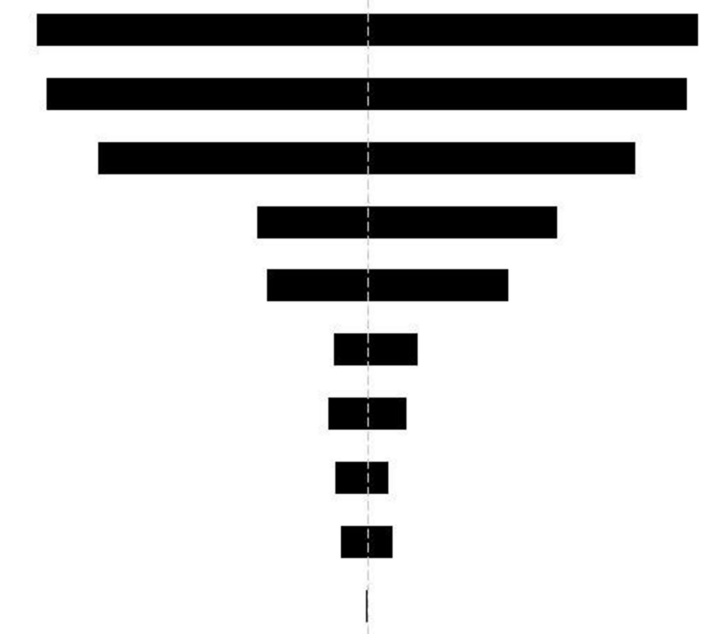

The cost of PD for the Placbo group

The cost of Olaprib

The cost of PD for the Olaprib group

The utility of PFS

The utility of PD

The duration of PFS for the Olaprib group

The cost of test for the Olaprib group

The duration of PFS for the Placbo group

The cost of test for the Placbo group

The cost of AEs for the Olaprib group

The cost of AEs for the Placbo group

\section{$25000 \quad 30000 \quad 35000 \quad 40000 \quad 45000$}

Figure 3 Tornado diagram of one-way sensitivity analysis. This summarizes the results of one-way sensitivity analysis, listing influential parameters in descending order according to their effect on the ICER over the variation of each parameter value.

Abbreviations: c, cost; PFS, progression-free survival; PD, progressive disease; AE, adverse event.

\section{CE Acceptability Curve}

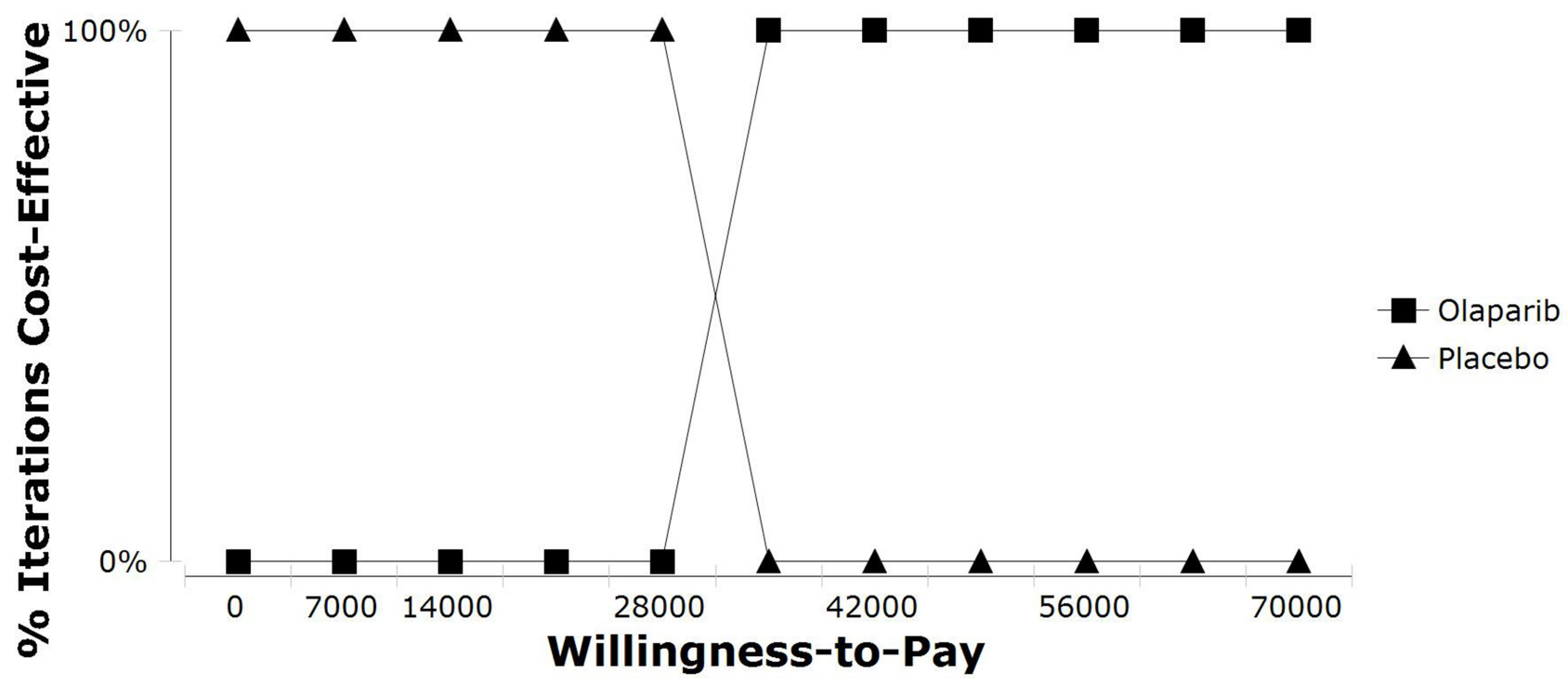

Figure 4 Cost-effectiveness acceptability curves. Cost-effectiveness acceptability curves show the probability of each treatment strategy being cost-effective at different WTP thresholds.

When we calculated the cost of olaparib according to the donation plan, the ICER decreased to $\$ 14,563.22$ per QALY, which was less than WTP. Due to the drug price negotiation mechanism in China, the prices of many effective, albeit expensive, treatments have decreased dramatically, especially those of anticancer drugs. In addition, these medicines 
for serious diseases, such as cancer, can be included in China's medical insurance reimbursement list after negotiations. Olaparib was included in the list of drugs for national drug negotiation in 2019, but olaparib for pancreatic cancer patients has not been added to the medical insurance list because of off-label use. We assumed that the fees for olaparib paid by metastatic pancreatic cancer patients with a germline BRCA mutation could be reimbursed up to approximately $70 \%$. The ICER of olaparib is estimated to be $\$ 6754.95$ per QALY and $\$ 22,371.50$ per QALY from the perspectives of patients and medical insurance, respectively.

WTP is a critical parameter in determining whether a drug is cost-effective. The National Institute of Health and Clinical Excellence (NICE) increased the costeffectiveness threshold for life-extending treatments for terminally ill patients. ${ }^{19}$ However, China did not specify which diseases could have the highest WTP, and many costeffectiveness studies among Chinese payers set WTP according to GDP per capita. Whether WTP needs to be adjusted for pancreatic cancer, which lacks effective treatment and has a low survival rate, is a question that needs to be considered. The ICERs associated with novel anticancer drugs may be unacceptably high in China. ${ }^{20-22}$ Given that there are no other effective drugs for the maintenance treatment of pancreatic cancer and the proportion of pancreatic cancer patients with BRCA mutations is very low, PFS improvement derived from maintenance olaparib can be viewed as a major therapeutic advance. Some measures should be taken to improve the cost-effectiveness of these novel anticancer drugs. Pharmaceutical companies or charities may provide assistance programs. Recently, China has introduced a series of legislative and policy reforms to relax administrative control over drug prices, and a national drug negotiations mechanism has been established. After national drug negotiations, olaparib will have an appropriate price discount and be included in medical insurance. The fees for olaparib paid by ovarian cancer, salpingocarcinoma or primary peritoneal cancer patients will be reimbursed in 2020. Although olaparib treatment for pancreatic cancer patients with BRCA mutations is off label in China, olaparib was approved as maintenance treatment in adult patients with deleterious or suspected deleterious germline BRCA-mutated metastatic pancreatic adenocarcinoma by the United States Food and Drug Administration as an orphan drug in 2019. Due to the lack of an effective treatment for the small number of pancreatic cancer patients with BRCA mutations, olaparib (Lynparza) has been granted an orphan drug designation in Japan and the
United States. The economic evaluation of orphan drugs is significantly different from that of ordinary drugs. Drug evaluation committees in some countries, such as NICE committees, approve orphan drugs with high WTP because of their social welfare value. ${ }^{23,24}$ Therefore, the government can consider including olaparib in medical insurance for this small patient population. Both the application of donation policy and increasing WTP can improve the economy of olaparib. Governments could try to work together with pharmaceutical companies and charities to alter olaparib's affordability.

Our study inevitably has weaknesses because our model relied on a phase III trial rather than real-world practice. The POLO trial is a large-sample and high-quality phase III trial, but maintenance olaparib was not a standard treatment for pancreatic cancer before the POLO trial. Therefore, we cannot conduct a cost-effectiveness analysis based on real-world research at present. There is no health utility value of pancreatic cancer for Chinese patients, and the POLO trial did not report health utility values of the PFS and PD states. Maintenance olaparib did not compromise the quality of life compared with placebo in the POLO trial, ${ }^{11,14}$ so we assumed the utility scores to be similar between the two groups in the same health state and used the health utility value of nonAsian patients from published studies in our model. In addition, the POLO trial did not provide enough detailed information on sequential therapy, and we assumed that the costs for AEs and testing in the PD state were the same and did not include these costs when calculating the cost of the PD state. According to the sensitivity analysis, the cost for the PD state for both groups had a major impact on the ICER. We estimated the cost of the PD state based mainly on the price of anticancer drugs. With the pilot reform policy of centralized drug procurement, the prices of an increasing number of drugs can be reduced in China. According to the one-way sensitivity analysis, a reduction in the cost of the PD state and olaparib could change olaparib's affordability. In other words, a reduction in drug prices would alter our result, so we need to update the cost-effectiveness analysis according to the fluctuation in drug price over time.

To our knowledge, this is the first study to evaluate the cost-effectiveness of olaparib as maintenance treatment for metastatic pancreatic cancer. Because of the high price of olaparib, maintenance olaparib for BRCA-mutated metastatic pancreatic cancer was not cost-effective compared with placebo. Assumptions are inevitable in the model method, and many factors influenced the ICER, but sensitivity analysis was conducted to reduce uncertainty. The present results are 
potentially useful for health-care decision-making. After more patients with BRCA-mutated metastatic pancreatic cancer use olaparib as maintenance treatment, real-world studies are needed to verify its efficacy, safety and economy.

\section{Ethical Approval}

This article does not contain any studies with human participants or animals performed by any of the authors.

\section{Author Contributions}

All authors made a significant contribution to the work reported, whether that is in the conception, study design, execution, acquisition of data, analysis and interpretation, or in all these areas; took part in drafting, revising or critically reviewing the article; gave final approval of the version to be published; have agreed on the journal to which the article has been submitted; and agree to be accountable for all aspects of the work.

\section{Funding}

This work was supported by the 1.3.5 Project for Disciplines of Excellence, West China Hospital, Sichuan University (No. ZYJC18010).

\section{Disclosure}

The authors declare they have no conflicts of interest.

\section{References}

1. Bray F, Ferlay J, Soerjomataram I, Siegel RL, Torre LA, Jemal A. Global cancer statistics 2018: GLOBOCAN estimates of incidence and mortality worldwide for 36 cancers in 185 countries. CA Cancer J Clin. 2018;68(6):394-424.

2. Siegel RL, Miller KD, Jemal A. Cancer statistics, 2020. CA Cancer J Clin. 2020;70(1):7-30.

3. Rawla P, Sunkara T, Gaduputi V. Epidemiology of pancreatic cancer: global trends, etiology and risk factors. World $J$ Oncol. 2019;10 (1):10-27. doi:10.14740/wjon1166

4. Siegel RL, Miller KD, Jemal A. Cancer statistics, 2019. CA Cancer J Clin. 2019;69(1):7-34.

5. Rahib L, Smith BD, Aizenberg R, Rosenzweig AB, Fleshman JM, Matrisian LM. Projecting cancer incidence and deaths to 2030: the unexpected burden of thyroid, liver, and pancreas cancers in the United States. Cancer Res. 2014;74(11):2913-2921. doi:10.1158/ 0008-5472.CAN-14-0155

6. Ghiorzo P. Genetic predisposition to pancreatic cancer. World $J$ Gastroenterol. 2014;20(31):10778-10789. doi:10.3748/wjg.v20. i31.10778

7. Holter S, Borgida A, Dodd A, et al. Germline BRCA mutations in a large clinic-based cohort of patients with pancreatic adenocarcinoma. J Clin Oncol. 2015;33(28):3124-3129. doi:10.1200/JCO.2014.59.7401

8. Friedenson B. BRCA1 and BRCA2 pathways and the risk of cancers other than breast or ovarian. Med Gen Med. 2005;7(2):60
9. Moore K, Colombo N, Scambia G, et al. Maintenance olaparib in patients with newly diagnosed advanced ovarian cancer. $N$ Engl $J$ Med. 2018;379(26):2495-2505. doi:10.1056/NEJMoa1810858

10. Pujade-Lauraine E, Ledermann JA, Selle F, et al. Olaparib tablets as maintenance therapy in patients with platinum-sensitive, relapsed ovarian cancer and a BRCA1/2 mutation (SOLO2/ENGOT-Ov21): a double-blind, randomised, placebo-controlled, Phase 3 trial. Lancet Oncol. 2017;18(9):1274-1284. doi:10.1016/S1470-2045(17)30469-2

11. Golan T, Hammel P, Reni M, et al. Maintenance olaparib for germline BRCA-mutated metastatic pancreatic cancer. $N$ Engl $J$ Med. 2019;381(4):317-327.

12. National Comprehensive Cancer Network. Pancreatic adenocarcinoma version 1.2020.; 2020. Available from: https://www.ncen.org/ professionals/physician_gls/pdf/pancreatic.pdf. Accessed November 28, 2019.

13. Hoyle MW, Henley W. Improved curve fits to summary survival data: application to economic evaluation of health technologies. BMC Med Res Methodol. 2011;11:139. doi:10.1186/1471-2288-11-139

14. Hammel P, Kindler HL, Reni M, et al. Health-related quality of life in patients with a germline BRCA mutation and metastatic pancreatic cancer receiving maintenance olaparib. Ann Oncol. 2019;30 (12):1959-1968. doi:10.1093/annonc/mdz406

15. Romanus D, Kindler HL, Archer L, et al. Does health-related quality of life improve for advanced pancreatic cancer patients who respond to gemcitabine? Analysis of a randomized phase III trial of the cancer and leukemia group B (CALGB 80303). J Pain Symptom Manage. 2012;43(2):205-217. doi:10.1016/j.jpainsymman.2011.09.001

16. Eichler HG, Kong SX, Gerth WC, Mavros P, Jonsson B. Use of cost-effectiveness analysis in health-care resource allocation decision-making: how are cost-effectiveness thresholds expected to emerge? Value Health. 2004;7(5):518-528. doi:10.1111/j.15244733.2004.75003.x

17. Smith HJ, Walters Haygood CL, Arend RC, Leath CA 3rd, Straughn JM Jr. PARP inhibitor maintenance therapy for patients with platinum-sensitive recurrent ovarian cancer: a cost-effectiveness analysis. Gynecol Oncol. 2015;139(1):59-62. doi:10.1016/j.ygyno. 2015.08.013

18. Saito S, Nakazawa K, Nagahashi M, Ishikawa T, Akazawa K. Costeffectiveness of BRCA1/2 mutation profiling to target olaparib use in patients with metastatic breast cancer. Per Med. 2019;16(6):439-448. doi:10.2217/pme-2018-0141

19. NICE. PMG19 Addendum A-Final amendments to the NICE technology appraisal processes and methods guides to support the proposed new Cancer Drugs Fund arrangements. London: NICE; 2016.

20. Zhan M, Zheng H, Xu T, Yang Y, Li Q. Cost-effectiveness analysis of additional bevacizumab to pemetrexed plus cisplatin for malignant pleural mesothelioma based on the MAPS trial. Lung Cancer. 2017;110:1-6. doi:10.1016/j.lungcan.2017.05.012

21. Li LY, Wang H, Chen X, Li WQ, Cui JW. First-line atezolizumab plus chemotherapy in treatment of extensive small cell lung cancer: a cost-effectiveness analysis from China. Chin Med J. 2019;132 (23):2790-2794. doi:10.1097/CM9.0000000000000536

22. Liu Q, Luo X, Peng L, et al. Nivolumab versus docetaxel for previously treated advanced non-small cell lung cancer in China: a cost-effectiveness analysis. Clin Drug Investig. 2019.

23. Hyry HI, Stern AD, Cox TM, Roos JC. Limits on use of health economic assessments for rare diseases. QJM. 2014;107 (3):241-245. doi:10.1093/qjmed/hcu016

24. Cowles E, Marsden G, Cole A, Devlin N. A review of NICE methods and processes across health technology assessment programmes: why the differences and what is the impact? Appl Health Econ Health Policy. 2017;15(4):469-477. doi:10.1007/s40258-017-0309-y 


\section{Publish your work in this journal}

Cancer Management and Research is an international, peer-reviewed open access journal focusing on cancer research and the optimal use of preventative and integrated treatment interventions to achieve improved outcomes, enhanced survival and quality of life for the cancer patient.
The manuscript management system is completely online and includes a very quick and fair peer-review system, which is all easy to use. Visit http://www.dovepress.com/testimonials.php to read real quotes from published authors.

Submit your manuscript here: https://www.dovepress.com/cancer-management-and-research-journal 